\title{
Research Article \\ Effect of Fiber Waviness on Tensile Strength of a Flax-Sliver-Reinforced Composite Material
}

\author{
Taweesak Piyatuchsananon, ${ }^{1}$ Akira Furuya, ${ }^{1}$ Baosheng Ren, ${ }^{2}$ and Koichi Goda ${ }^{3}$ \\ ${ }^{1}$ Graduate School of Science and Engineering, Yamaguchi University, Tokiwadai, Ube 755-8611, Japan \\ ${ }^{2}$ School of Material Science and Engineering, University of Jinan, Jinan, Shandong 250022, China \\ ${ }^{3}$ Department of Mechanical Engineering, Yamaguchi University, Ube 755-8611, Japan \\ Correspondence should be addressed to Koichi Goda; goda@yamaguchi-u.ac.jp
}

Received 11 January 2015; Accepted 2 March 2015

Academic Editor: P. J. Schubel

Copyright (C) 2015 Taweesak Piyatuchsananon et al. This is an open access article distributed under the Creative Commons Attribution License, which permits unrestricted use, distribution, and reproduction in any medium, provided the original work is properly cited.

\begin{abstract}
Recently, a composite material made from natural fibers and biodegradable resin, "green composite," is attracting attention as an alternative composite material for the replacement of glass fiber-reinforced plastics. Plant-based natural fibers such as kenaf and flax have already been used as composite reinforcement materials because they are more environmentally friendly and costless fibers than artificial fibers. A problem of using natural fibers is the fiber waviness, which affects the tensile properties. Fiber waviness is fluctuation in the fiber orientation that is inherent in the sliver morphology of plant-based natural fibers. This study was conducted to clarify the relation between quantified parameters of fiber waviness and a composite's tensile strength. First, the fiber orientation angles on a flax-sliver-reinforced composite were measured. Then the angle distribution was quantified through spatial autocorrelation analysis methods: Local Moran's I and Local Geary's $c$. Finally, the relation between the resultant tensile strength and quantified parameters was discussed.
\end{abstract}

\section{Introduction}

Strong demand for the use of composite materials is increasing today because high strength and stiffness, as well as low density, are needed to reduce energy consumption in aviation and automotive transport industries. The use of artificial fiber-reinforced composite materials, such as glass fiberreinforced plastics (GFRP) and carbon fiber-reinforced plastics (CFRP), is effective to meet these demands, but disposal difficulties that arise after their use have surfaced as an environmental problem. Therefore, many researchers have strived to develop biodegradable renewable composite materials and various production methods for the materials that will widen their practical availability [1-5]. Plant-based natural fibers, such as flax, hemp, ramie, jute, kenaf, curaua, and bamboo, are expected for use as the composite reinforcement materials, but these are often used as short fibers, as seen in injection-molded products. Long fibers are generally known to exhibit load-bearing potential in a matrix material as compared to short fibers. Before spinning process, plant-based natural fibers are often supplied as long fibers called "slivers." When this form is successfully prepared with resin, slivers can be applied as a semifinished composite material in prepregs $[5,6]$. One of the problems in preparing slivers is their fiber waviness, which is fluctuation in the fiber orientation inherent in plant-based natural fibers as well as synthetic fibers. Such waviness often engenders a decrease in the mechanical properties of the composites. It was pointed out in the model proposed by Hsiao and Daniel [7] that the decrease in elastic properties of a unidirectional carbon/epoxy composite resulted from the fiber waviness. Karami and Garnich [8] used a finite element micromechanical model to predict the effects of periodic and localized fiber waviness on carbon fiber reinforcement. In these papers, however, the fiber waviness was assumed as a deterministic shape such as a sine curve. In order to take the stochastic wavy effect of sliver into account, Ren et al. $[9,10]$ quantified the fibre orientation fluctuation in curaua-sliver- and flax-sliver-reinforced composite through one-dimensional and two-dimensional 


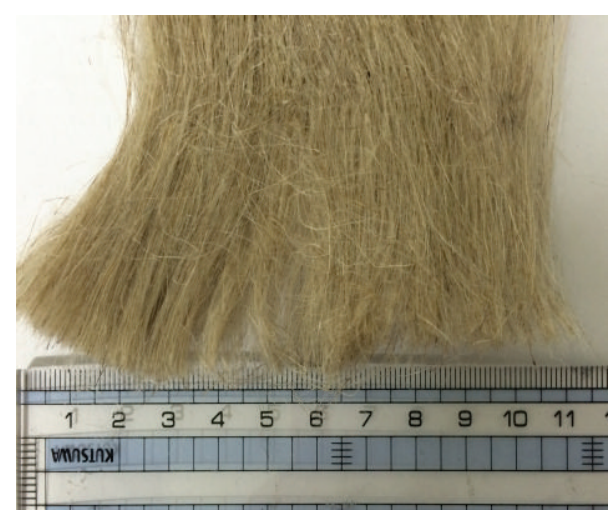

Figure 1: Flax sliver.

autocorrelations between fiber orientation angles on the composite surface. The quantified parameter expressing the degree of the fluctuation, called the "area ratio," was correlated with the composite tensile strength. However, the use of autocorrelations was intended to evaluate the quality of fiber orientation but not to express the degree of local disorder in fiber orientation. In general, stiffness is insensible for structural defects, but strength is sensible. Therefore, the local disorder in fiber orientation is of great interest for quantification through some statistical or stochastic analytical method. It is also expected that the quantified parameter shall play a role as a key indicator during quality inspection for produced prepregs.

Thus, the purpose of this study is to examine the effects of the fiber waviness on the tensile strength of a flax-sliverreinforced composite (in-plane tensile modulus). Resinpasted flax slivers were first compression molded, and then fiber orientation angles were measured on the surfaces of the resultant composite laminates. To quantify the degree of the disorder in fiber orientation, the measurement results were analyzed by Local Moran's I and Local Geary's c [11], which are measures of the representative spatial autocorrelation analyses. Quantification by the "area ratio" was optimized by seeking an appropriate threshold level of the measures, correlated closely with tensile strength data.

\section{Experimental}

2.1. Summary of Experimental Method. Since the present experimental results are based on the previous paper [10], in this section the experimental method is briefly introduced as follows.

Materials. Flax slivers, one of the representative natural fibers, were used as reinforcement as shown in Figure 1. The slivers were supplied from Teikoku Sen-i Co., Ltd., Japan. Biodegradable thermoplastic resin was used as the matrix, which was supplied from Miyoshi Oil and Fat Co., Ltd., Japan (product name: Randy PL-1000). The resin was supplied in a water emulsion containing microorder fine particles of approximately $5.0 \mu \mathrm{m}$ diameter. The Randy PL-1000 is made from plant-derived biodegradable resins. In this sense,

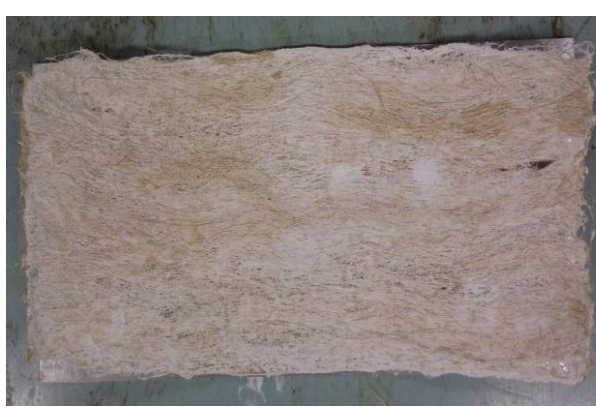

FIGURE 2: Resin-pasted sliver (preform).

the resultant composite is a "fully green composite." Typical physical and mechanical properties of these constituents are shown in Table 1.

Molding Process. First, the fibers on the front side were closed with resin and dried for 24 hours. Subsequently, another side (back side) was done with the same process as the front side. The thickness was 0.4 to $0.6 \mathrm{~mm}$, of which the range was enough to exhibit nearly the same fiber orientation at the surface and inside. Such resin-pasted slivers are called preforms or prepregs. Figure 2 shows the photograph of the preform. At each process, the weight was measured for calculation of the fiber content. After finishing preparation of the preforms, we cut them to $100 \mathrm{~mm} \times 100 \mathrm{~mm}$ and put two pieces into the mold. The mold was set at $150^{\circ} \mathrm{C}$ for $40 \mathrm{~min}$, and then the hydraulic press was set at $3 \mathrm{MPa}$ pressure. The temperature was reduced to room temperature at pressure of $3 \mathrm{MPa}$ for 24 hours. The fiber volume fractions $V_{f}$ of all fabricated composites were calculated using the following equation:

$$
V_{f}=1-\frac{W-W_{f}}{\rho_{m} V},
$$

where $W$ is the fabricated composite weight, $W_{f}$ is the flax fibre weight in the composite, $V$ is the fabricated composite volume, and $\rho_{m}$ is the biodegradable resin density.

Two methods were used to fabricate the composites: sheet lamination method (SLM) and direct method (DM). In $\mathrm{DM}$, the resin was pasted directly for the as-supplied slivers. This method is more appropriate for mass production than SLM, but products fabricated using DM include numerous disordered parts in the fiber orientation. In SLM, the sliver was combed carefully to form unidirectionally oriented fibers before resin pasting.

Tensile Tests. Tensile specimens were cut to $15 \mathrm{~mm}$ width from DM and SLM composite laminates of $100 \mathrm{~mm} \times 100 \mathrm{~mm}$ square, and then aluminum plates $(15 \mathrm{~mm} \times 15 \mathrm{~mm})$ were attached with epoxy adhesive to both ends of all composite specimens for tensile testing. To prevent stress concentration near aluminum plates during tensile testing, their edges were shaved to $45^{\circ}$. A strain gage was attached on the center of 
TABLE 1: Properties of the fibers and matrix $[12,13]$.

\begin{tabular}{lccccc}
\hline Material & Density $\left(\mathrm{Mg} / \mathrm{m}^{3}\right)$ & Fiber width $(\mu \mathrm{m})$ & Tensile strength $(\mathrm{MPa})$ & Fracture strain $(\%)$ & Young's modulus $(\mathrm{GPa})$ \\
\hline Randy PL-1000 & 1.20 & - & 32.5 & - & 3.8 \\
Flax fiber & 1.50 & $10-30$ & $600-1100$ & $1.5-2.4$ & $40-100$ \\
\hline
\end{tabular}

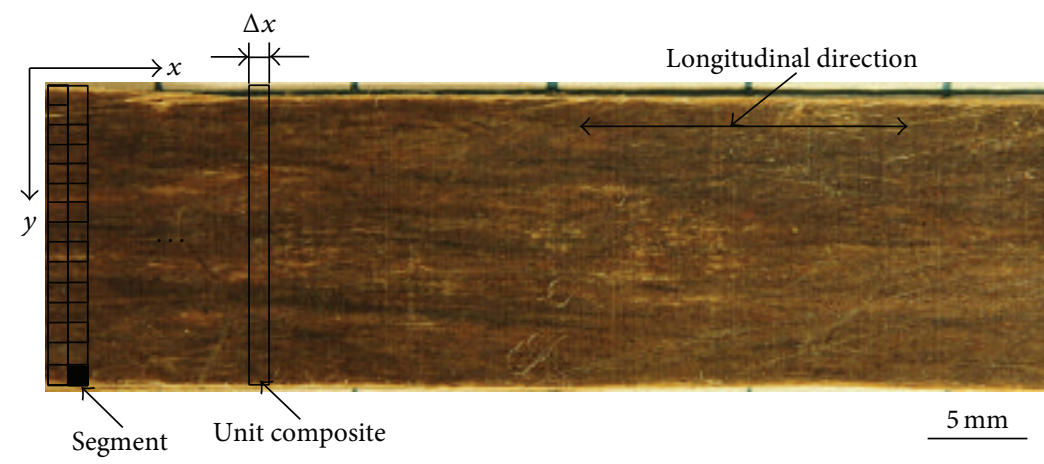

(a) Division into segment

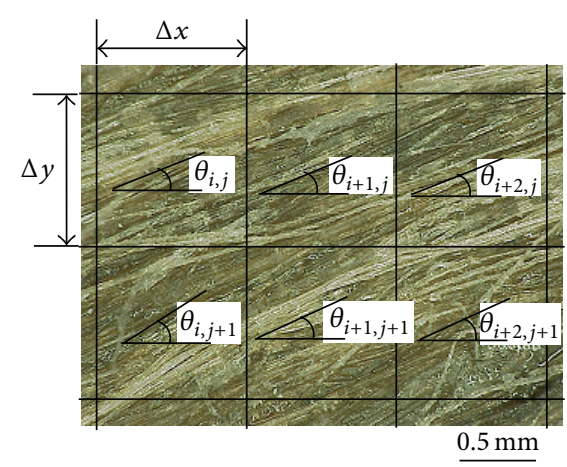

(b) Angle $\theta$ in a segment $(\Delta x=\Delta y=1 \mathrm{~mm})$

FIGURE 3: Measurement of fiber orientation angles on a flax-sliver-reinforced composite laminate.

specimens to measure uniaxial strain. Tensile tests were carried out using an Instron-type testing machine (Autograph IS-500; Shimadzu Corp.) with cross-head speed of $1 \mathrm{~mm} / \mathrm{min}$.

Angle Measurements. Fiber orientation angles are important data for determining the strength and stiffness of a composite with fiber waviness. In this measurement, the $x$-axis is the longitudinal direction and $y$-axis is the transverse direction. Specimens were divided into a small size as $1 \mathrm{~mm}$ for $\Delta x$ and $\Delta y$, which consist of 50 unit composites along the $x$-axis and one unit composite divided into 15 segments along the $y$ axis, as shown in Figure 3(a). The fiber orientation angle to the $x$-axis was measured in each segment of both sides, as shown in Figure 3(b). The total segments were 750 for each side. Image analysis software (Asahi Kasei Corp., Japan) was used for angle measurements.

2.2. Spatial Autocorrelation Analysis. In this study, two representative spatial autocorrelation analyses, Local Moran's $I$ and Local Geary's $c$ [11], were used to define the degree of local disorder of fiber orientation on tensile strength.

2.2.1. Local Moran's I. Moran's I was created by Moran [11] as a typical tool of spatial autocorrelation to analyze the deviation from the average. Local Moran's $I$ is given as

$$
I\left(\theta_{i}\right)=\frac{\left(\theta_{i}-\bar{\theta}\right)}{(1 / n) \sum_{i=1}^{n}\left(\theta_{i}-\bar{\theta}\right)^{2}} \sum_{j=1, j \neq i}^{n} w_{i j}(d)\left(\theta_{j}-\bar{\theta}\right)
$$

where $w_{i j}(d)$ is the weight function of the pair samples in distance $d$ between the pair points, $i$ th and $j$ th locations, given as

$$
w_{i j}(d)=\left\{\left(x_{i}-x_{j}\right)^{2}+\left(y_{i}-y_{j}\right)^{2}\right\}^{-1 / 2},
$$

where $x_{i}$ is $i$ th position of the $x$-axis and the range is $1-50 \mathrm{~mm}$. Also, $y_{i}$ is the $i$ th position of the $y$-axis, and the range is 1$15 \mathrm{~mm} . \theta_{i}$ and $\theta_{j}$ are the angle data at the $i$ th and $j$ th positions, respectively. $\bar{\theta}$ is the average angle. Hereinafter, Local Moran's $I$ is denoted as LM- $I$. LM- $I$ varies between -1 and +1 . If LM- $I$ approaches +1 , then the angle at this location is more largely far from the average, but similar to the neighbor's angles in their deviation from the average. On the other hand, if LM-I tends to approach -1 , then the angle at this location is also higher or lower than the average. But, the sign is different from the neighbor angles. When LM-I tends to approach 0 , the angle at this location is similar to the average. Theoretically, when LM- $I$ is either much higher or lower than 0 , then the fiber orientation angle is significantly different from the average. Consequently, such LM-I points, if gathered locally, could form a large disordered area in fiber orientation.

2.2.2. Local Geary'sc. Local Geary's $c$ is another typical spatial autocorrelation to analyze a deviation from the surroundings. Local Geary's $c$ is shown as

$$
c\left(\theta_{i}\right)=\frac{1}{(1 / n) \sum_{i=1}^{n}\left(\theta_{i}-\bar{\theta}\right)^{2}} \sum_{j=1, j \neq i}^{n} w_{i j}(d)\left(\theta_{i}-\theta_{j}\right)^{2} .
$$

Local Geary's $c$ is hereinafter denoted as LG-c. LG-c varies between 0 and 1 . When $L G-c$ tends to approach 0 , the angle at this location is similar to the neighbor angles. In contrast, when LG- $c$ tends to approach 1, the angle at this location differs from the sign of neighbor angles or is much higher than the neighbor's angles in absolute value. Consequently, such points can be disordered parts in fiber orientation.

2.3. Calculation of Area Ratio. To quantify the degree of local disorder in fiber orientation, we defined the "area ratio" which can be obtained from the contour maps of LM-I and LG-c. 
TABLE 2: Fiber orientation angles and mechanical properties of flax-sliver-reinforced green composites [10].

\begin{tabular}{|c|c|c|c|c|c|c|c|c|}
\hline \multirow{3}{*}{$\begin{array}{l}\text { Production } \\
\text { method }\end{array}$} & \multirow{3}{*}{$\begin{array}{l}\text { Sample } \\
\text { number }\end{array}$} & \multirow{3}{*}{$\begin{array}{c}\text { Fiber volume } \\
\text { fraction }\end{array}$} & \multicolumn{4}{|c|}{ Specimens } & \multirow{3}{*}{$\begin{array}{l}\text { Tensile strength and } \\
\text { (standard deviation) } \\
(\mathrm{MPa})\end{array}$} & \multirow{3}{*}{$\begin{array}{l}\text { Young's } \\
\text { modulus } \\
(\mathrm{GPa})\end{array}$} \\
\hline & & & Side A (l & ower angle) & Side B (h & iigher angle) & & \\
\hline & & & Avg. angle $\left({ }^{\circ}\right)$ & S.D. of angles $\left({ }^{\circ}\right)$ & Avg. angle $\left({ }^{\circ}\right)$ & S.D. of angles $\left({ }^{\circ}\right)$ & & \\
\hline SLM & - & 0.72 & 0 & - & 0 & - & 238 & 40.5 \\
\hline \multirow{11}{*}{$\mathrm{DM}$} & $<1>$ & 0.55 & 1.99 & 3.54 & 3.30 & 4.28 & 132 & 22.7 \\
\hline & $<2>$ & 0.55 & 2.62 & 5.22 & 4.19 & 4.03 & 224 & 26.7 \\
\hline & $<3>$ & 0.55 & 5.14 & 4.06 & 5.55 & 3.18 & 158 & 29.6 \\
\hline & $<4>$ & 0.55 & 3.05 & 3.22 & 8.76 & 4.18 & 211 & 27.6 \\
\hline & $<5>$ & 0.65 & 1.74 & 3.73 & 2.85 & 2.66 & 158 & 23.3 \\
\hline & $<6>$ & 0.65 & 2.10 & 2.62 & 3.07 & 3.48 & 203 & 26.4 \\
\hline & $<7>$ & 0.65 & 1.61 & 2.33 & 2.20 & 2.32 & 153 & 27.5 \\
\hline & $<8>$ & 0.65 & 2.08 & 3.47 & 3.76 & 2.95 & 170 & 28.0 \\
\hline & $<9>$ & 0.65 & 1.45 & 3.72 & 5.26 & 4.77 & 173 & 26.8 \\
\hline & $<10>$ & 0.65 & 0.76 & 3.50 & 4.55 & 3.90 & 168 & 28.8 \\
\hline & Avg. & 0.61 & 2.35 & 3.50 & 4.35 & 3.60 & $175(28.81)$ & 26.8 \\
\hline
\end{tabular}

The number of SLM specimens was seven. Avg.: Average, S.D.: Standard deviation.

In this quantification, we used two image analysis programs; first "Graph R221" was used for replotting the above contour maps to binary images (black and white areas), and next "Azo R235" was used to calculate the area ratios of the black (risky areas) and white (nonrisky areas). The meaning of "risky area" is described in the next section.

\section{Results and Discussion}

3.1. Tensile Properties and Fiber Orientation Angle. Table 2 shows results of tensile tests. The fiber volume fraction of DM specimens is lower than that of SLM specimens because of the fiber waviness. It was confirmed from the angle measurement that DM specimens scattered in a wide range from positive twenty degrees to negative twenty degrees. Typical contour maps of fiber orientation angles are shown in Figures 4(a) and 4(b), in which the standard deviations are largely different. The former specimen has a relatively large positive angle distribution in the upper left-side part. On the other hand, the latter shows a uniform angle distribution. As known from Table 2, any tensile strength and Young's modulus of DM specimens are lower than those of SLM specimens. It is considered that such degradation is caused by the fiber waviness, as well as the lower fiber volume fraction.

As described in earlier papers $[9,10]$, the Young's modulus was well correlated with the average fiber orientation angle, which is recognized as an average property of fiber waviness. On the other hand, tensile strength was not correlated with it, because tensile strength is a mechanical property sensitive to local structural defects. It was concluded in the paper [10], however, that the degree of increase in tensile strength was correlated with the area ratio of well-oriented fibers, which was analyzed from two-dimensional autocorrelation between two arbitrary regions of $7 \times 15$ segments. When the area ratio was larger, the tensile strength tended to be higher. But this area ratio did not express the degree of local disorder in fiber orientation. In the next section, thus, we focus on quantification of the degree of local disorder.

3.2. Spatial Analysis. The contour maps of LM-I and LG-c are shown in Figures 5 and 6, in which the specimens are the same as those of Figure 4. In Figure 5, the large positive value segments are in nearly the same positions with the high angle distribution in absolute value in Figure 4. In comparison with the fractured specimens, we confirmed that the crack line tended to pass the positions of high coefficient of two methods. Thus, we call such positions the "risky area". In many specimens, the risky areas of both LM-I and LG-c included one part of the crack line.

The segments with larger LM- $I$ in absolute value would be loaded in shear and would suffer from premature damage, because many average angles in the specimens are relatively close to $0^{\circ}$, which are loaded in tension. If such premature damage is accumulated in the specimen, then it would cause premature tensile fracture, leading to low tensile strength. Therefore, the aggregate of such segments is risky from the viewpoint of materials reliability engineering. As shown in Figure 5, the scale of positive LM- $I$ is large and that of negative LM- $I$ is small. This is because a bunch of wavy fibers is larger in scale than the segment size, and its angles are measured as the same sign on the segments next to each other. In contrast, the segments with opposite signs in neighbors, which show negative LM- $I$, are only a few and are not major in scale. The scale of high LG-c values in Figure 6 is also small, as compared to that of positive LM- $I$ values in Figure 5. One reason is the same as the cause of negative value scale in LM-I. Another is considered such that, even if the neighbors have the same sign, then LG-c can approach unity on the condition that there is a large difference between their angles. But, the scale could not extend more than a single segment size, because of a wavy flow structure of sliver. In this case, also, the segment with larger angle in absolute value would be loaded in shear. Thus, although there is a difference in scale, the areas 


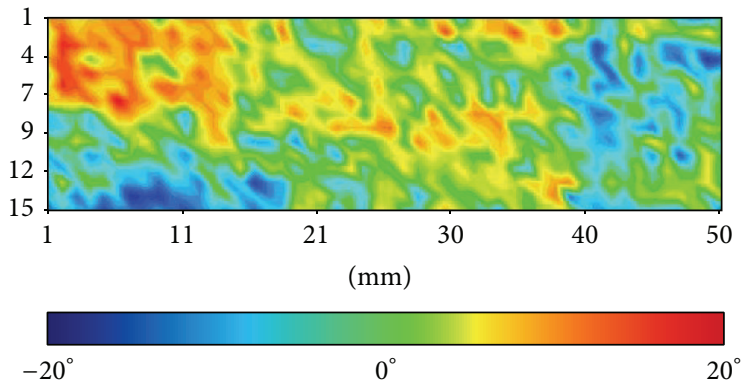

(a) Specimen $1 \mathrm{~A}\left(\bar{\theta}: 3.30^{\circ}\right.$, standard deviation: $\left.4.28^{\circ}\right)$

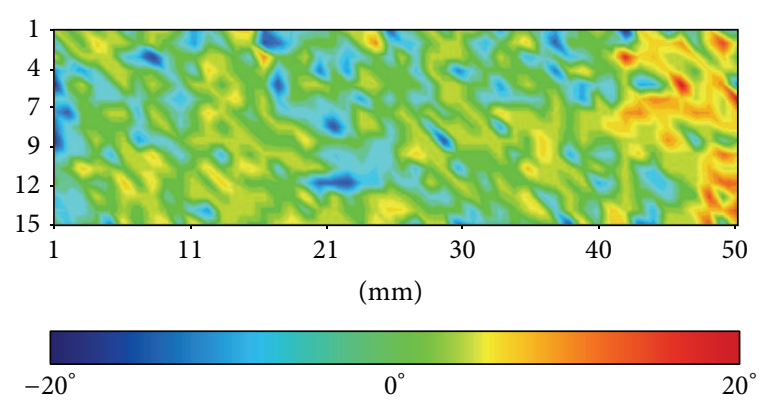

(b) Specimen $7 \mathrm{~A}\left(\bar{\theta}: 1.61^{\circ}\right.$, standard deviation: $\left.2.33^{\circ}\right)$

FIGURE 4: Contour map of fiber orientation angle distribution.
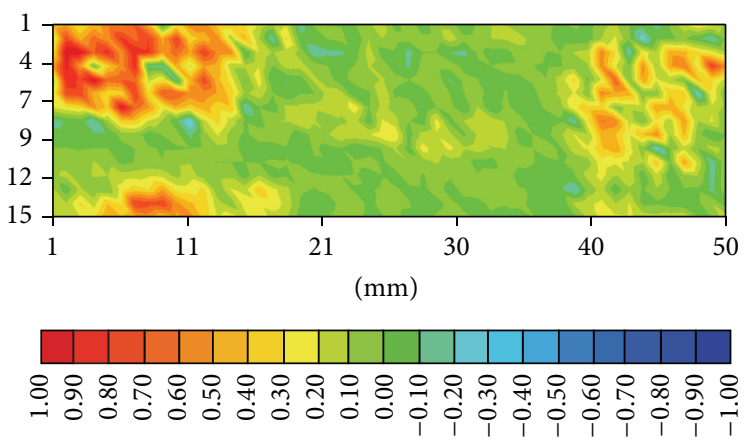

(a) Specimen $1 \mathrm{~A}$

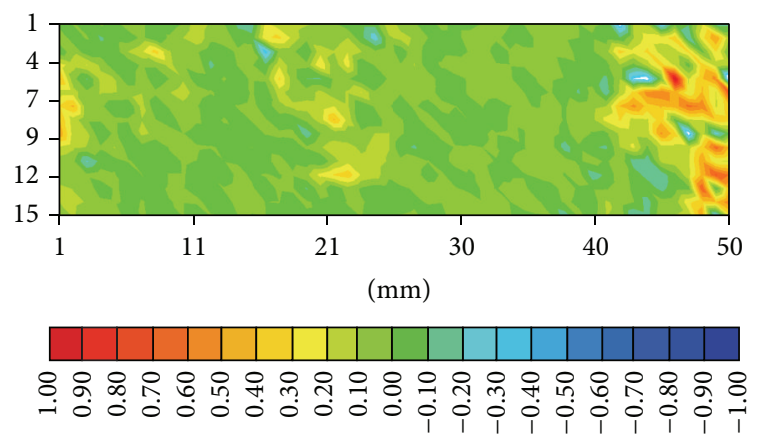

(b) Specimen $7 \mathrm{~A}$

FIgURE 5: Contour maps of Local Moran's I distribution.
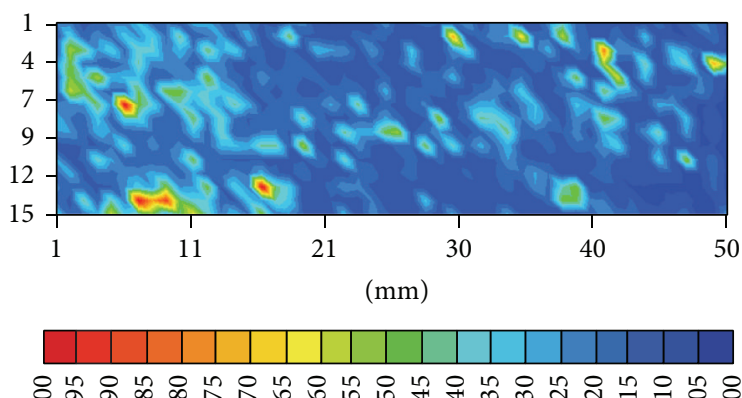

는 운

(a) Specimen number $1 \mathrm{~A}$

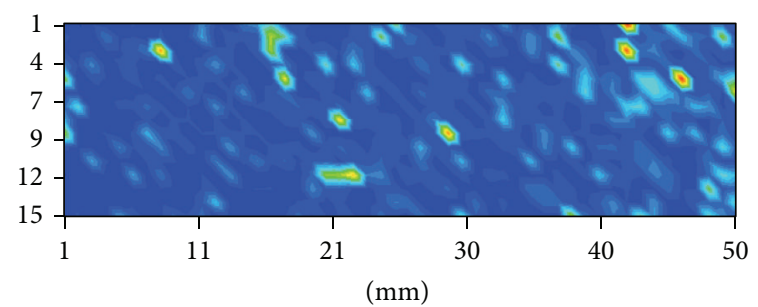

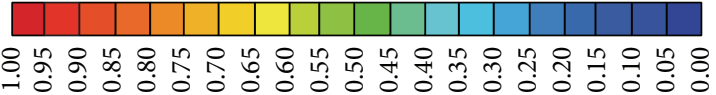

(b) Specimen number $7 \mathrm{~A}$

FIGURE 6: Contour maps of Local Geary's $c$ distribution.

with large positive LM- $I$, negative LM- $I$, and large LG- $c$ are equivalent to disordered parts in fiber orientation and may be correlated with tensile strength.

3.3. Area Ratio. Figure 7 shows LM-I binary images of specimens $1 \mathrm{~A}$ and $7 \mathrm{~A}$, in which the areas consisting of LM- $I$ higher than 0.65 or lower than -0.15 were temporarily selected. The numerical values, 0.65 and -0.15 , are so-called threshold levels, which divide the areas into white and black. In Figure 7, the percentage of black area ratio was $6.80 \%$ (positive side $=4.80 \%$, negative side $=2.00 \%$ ) for specimen $1 \mathrm{~A}$ and $3.47 \%$ (positive side $=0.67 \%$, negative side $=2.80 \%$ ) for specimen $7 \mathrm{~A}$. In these samples, the range of negative values was designated more widely than that of positive value, because the segments with negative LM- $I$ values are not so major but present the possibility of causing premature damage in shear, as mentioned in Section 3.2. Thus, wide range negative values and high positive values correspond to risky areas.

Regarding binary images of LG-c, the same samples as Figure 7 are shown in Figure 8. For these images, LG-c areas higher than 0.35 were selected. The value of 0.35 is a temporal threshold level. The percentages of black area ratio were $12.80 \%$ for specimen $1 \mathrm{~A}$ and $5.33 \%$ for specimen $7 \mathrm{~A}$, respectively. As is easily known, when the threshold levels of LM-I and LG-c are changed, the area ratios accordingly 

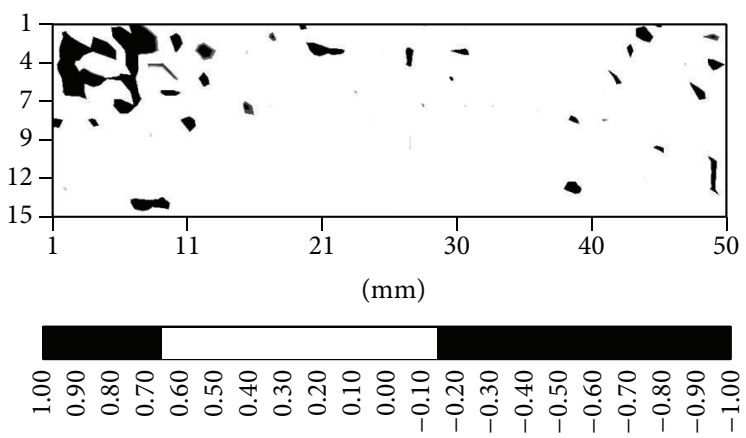

(a) Specimen $1 \mathrm{~A}$
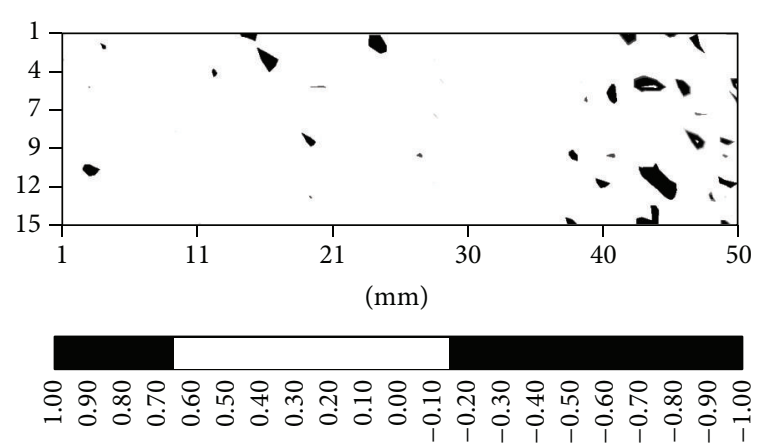

(b) Specimen $7 \mathrm{~A}$

FIGURE 7: Binary images of Local Moran's $I$ distribution (threshold levels: positive LM- $I=0.65$ and negative LM- $I=-0.15$ ).

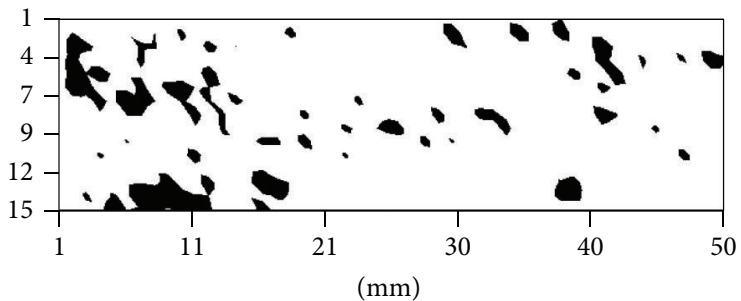

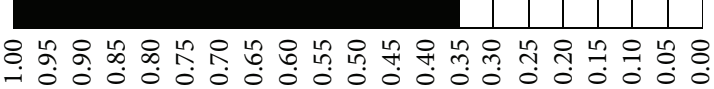

(a) Specimen $1 \mathrm{~A}$
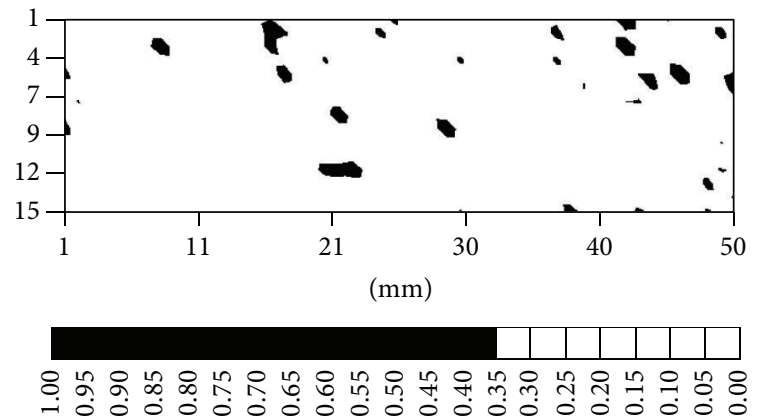

(b) Specimen 7A

FIgURE 8: Binary images of Local Geary's $c$ distribution (threshold level: LG-c $=0.35$ ).

change. We consider that, if appropriate threshold level(s) are given, then some optimal area ratio correlated with tensile strength exists for each specimen. This is because the ratio of segments suffering from shear damages during tensile loading should be related closely with the area ratio. In the next section, thus, the relation between area ratio and tensile strength is investigated.

3.4. Relation between Area Ratio and Tensile Strength. To investigate the correlation between the area ratio and tensile strength, normalized tensile strength data were plotted as a function of area ratio, as shown in Figures 9 and 10, where each tensile strength value was normalized at the same fiber volume fraction. That is to say, the tensile strengths were normalized by dividing the measured strength by the fiber volume fraction $V_{f}$ and then multiplying it by 0.72 , corresponding to $V_{f}$ of SLM specimen. In Figure 9, it appears that the normalized strength is correlated with the area ratio. The correlation coefficient in Figure 9 was calculated as -0.618 when setting the threshold levels at 0.65 (positive LM-I) and -0.15 (negative LM-I). The value of -0.618 is not so strong but presents an intermediate strong correlation. This means that if many segments in a specimen are distributed with LM- $I$ values higher or lower than the above threshold levels, its tensile strength tends to be lowered. It also means that a rough value of tensile strength can be estimated through the least-squares regression line between area ratio and tensile

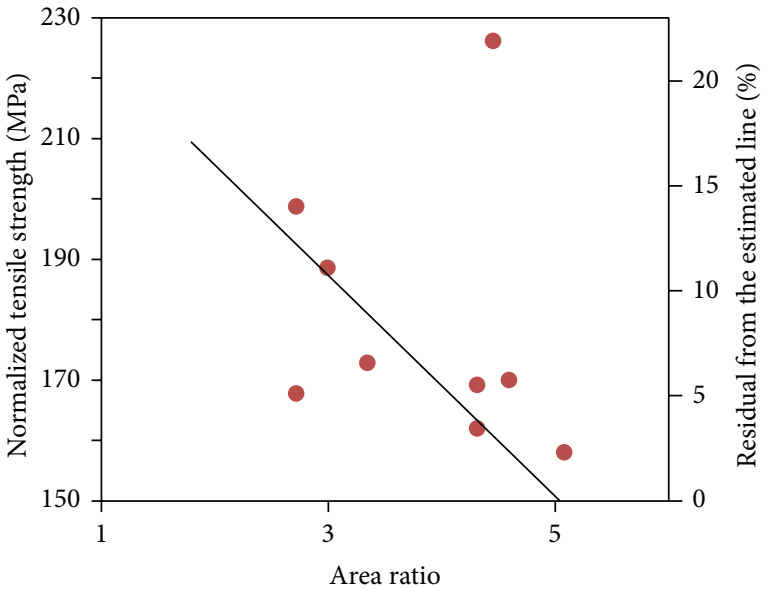

FIGURE 9: Area ratio dependence on normalized tensile strength (threshold levels: positive LM- $I=0.65$ and negative LM- $I=-0.15$ ).

strength. In contrast, the correlation coefficient between the area ratio and tensile strength in Figure 10 was only -0.117 , when choosing 0.35 as a threshold level. The value of -0.117 signifies weak correlation. This result implies that 0.35 is not appropriate as a threshold level or that Local Geary's $c$ does not match correlation with tensile strength. To find the optimal threshold levels of LM-I and LG-c, the negative 


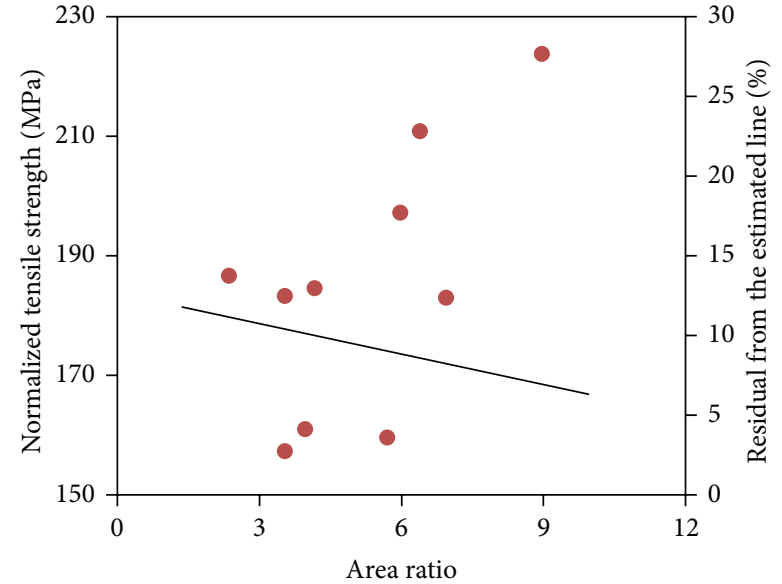

Figure 10: Area ratio dependence on normalized tensile strength (threshold level: LG- $c=0.35$ ).

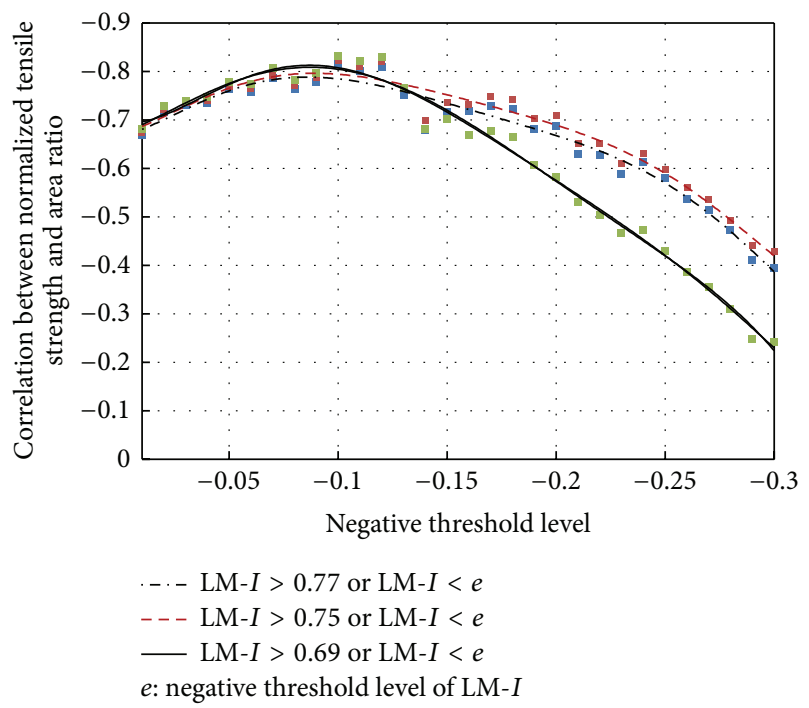

FIGURE 11: Correlation coefficients between LM-I area ratio and normalized tensile strength versus negative threshold level.

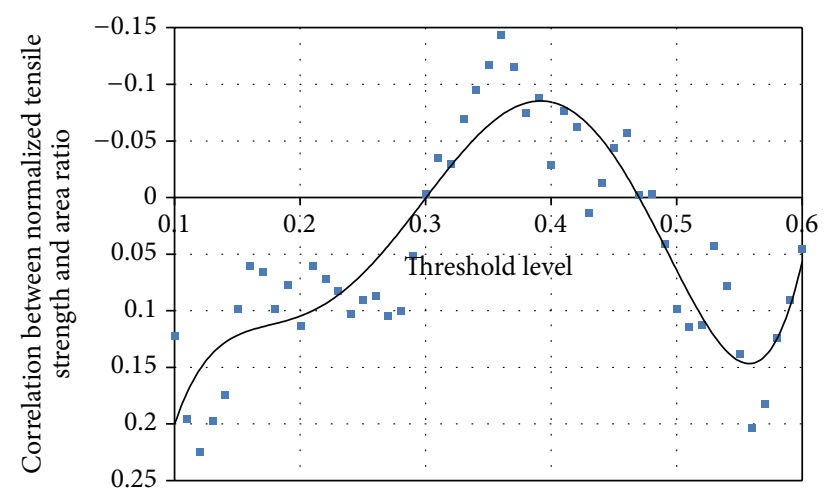

FIGURE 12: Correlation coefficients between LG-c area ratio and normalized tensile strength versus threshold level. threshold level of LM- $I$ was assigned in the range of -0.01 to -0.3 , and the threshold level of LG-c was in the range of 0.1 to 0.60 . For LM- $I$, the positive threshold level was also chosen as $0.69,0.75$, or 0.77 .

The results are shown in Figures 11 and 12. Figure 11 shows that the optimal threshold levels of LM-I are 0.69 at the positive level and -0.10 at the negative level, and that of LG-c is 0.36 . At the former optimal threshold levels, the correlation coefficient between the area ratio and tensile strength was -0.832 . On the other hand, the highest correlation coefficient in Figure 12 was only -0.14 even at the optimal threshold level. The value of -0.832 denotes a strong negative correlation between the area ratio and tensile strength. In other words, tensile strength can be estimated to some degree using the least-squares regression line when setting the optimal threshold levels of LM-I. It is also expected that the present procedure is applied as an effective screening method for extracting low-quality prepregs at quality inspection.

\section{Conclusions}

This study clarified the effect of fiber waviness on the tensile strength of a flax-sliver-reinforced biodegradable resin matrix composite material. The fiber waviness was quantified using Local Moran's $I$ and Local Geary's $c$, each of which can express the degree of disorder in fiber orientation. Results show that Local Moran's $I$ is correlated well with tensile strength of the composite specimens when appropriate threshold levels are selected. On the other hand, Local Geary's $c$ is not well correlated with tensile strength exists for each specimen. Normally, finite element analysis is a well-known as a method for elucidating the mechanical behavior of composite materials, such as stress and strain distributions. Furthermore, finite element analysis is often extended to the fracture process simulation to predict strength properties. However, the method needs to use several math models to ascertain the solution that takes time and the advanced knowledge for understanding. However, this study using the spatial autocorrelation analysis has fewer equations and does not take a long time for the program code development. Thus, we conclude that the method proposed in this study is an effective tool for rough prediction of the tensile strength of natural-fiber-sliver-based composite materials.

\section{Conflict of Interests}

The authors declare that there is no conflict of interests regarding the publication of this paper.

\section{References}

[1] L. Liu, J. Yu, L. Cheng, and X. Yang, "Biodegradability of poly(butylene succinate) (PBS) composite reinforced with jute fibre," Polymer Degradation and Stability, vol. 94, no. 1, pp. 9094, 2009.

[2] S. Alix, S. Marais, C. Morvan, and L. Lebrun, "Biocomposite materials from flax plants: preparation and properties," Composites Part A: Applied Science and Manufacturing, vol. 39, no. 12, pp. 1793-1801, 2008. 
[3] P. Lodha and A. N. Netravali, "Characterization of stearic acid modified soy protein isolate resin and ramie fiber reinforced 'green' composites,' Composites Science and Technology, vol. 65, no. 7-8, pp. 1211-1225, 2005.

[4] S. Serizawa, K. Inoue, and M. Iji, "Kenaf-fiber-reinforced poly(lactic acid) used for electronic products," Journal of Applied Polymer Science, vol. 100, no. 1, pp. 618-624, 2006.

[5] A. Gomes, T. Matsuo, K. Goda, and J. Ohgi, "Development and effect of alkali treatment on tensile properties of curaua fiber green composites," Composites Part A: Applied Science and Manufacturing, vol. 38, no. 8, pp. 1811-1820, 2007.

[6] LINEO FLAXTAPE, http://www.lineo.eu/\#!products.

[7] H. M. Hsiao and I. M. Daniel, Elastic Properties of Composite with Fiber Waviness, Elsevier Science, 1996.

[8] G. Karami and M. Garnich, "Effective moduli and failure considerations for composites with periodic fiber waviness," Composite Structures, vol. 67, no. 4, pp. 461-475, 2005.

[9] B. Ren, K. Goda, and J. Noda, "Effects of fiber orientation angles and fluctuation on the stiffness and strength of sliver-based green composites," Journal of the Society of Materials Science, Japan, vol. 59, no. 7, pp. 567-574, 2010.

[10] B. Ren, T. Mizue, K. Goda, and J. Noda, "Effects of fluctuation of fibre orientation on tensile properties of flax sliver-reinforced green composites," Composite Structures, vol. 94, no. 12, pp. 3457-3464, 2012.

[11] M.-J. Fortin and M. Dale, Spatial Analysis, Cambridge University Press, 2014.

[12] Flax slivers, Teikoku Sen-I, Tokyo, Japan, http://www.teisen.co .jp.

[13] Miyoshi Oil \& Fat, Randy PL-1000, Miyoshi Oil \& Fat, Tokyo, Japan, 2012, http://www.miyoshi-yushi.co.jp. 

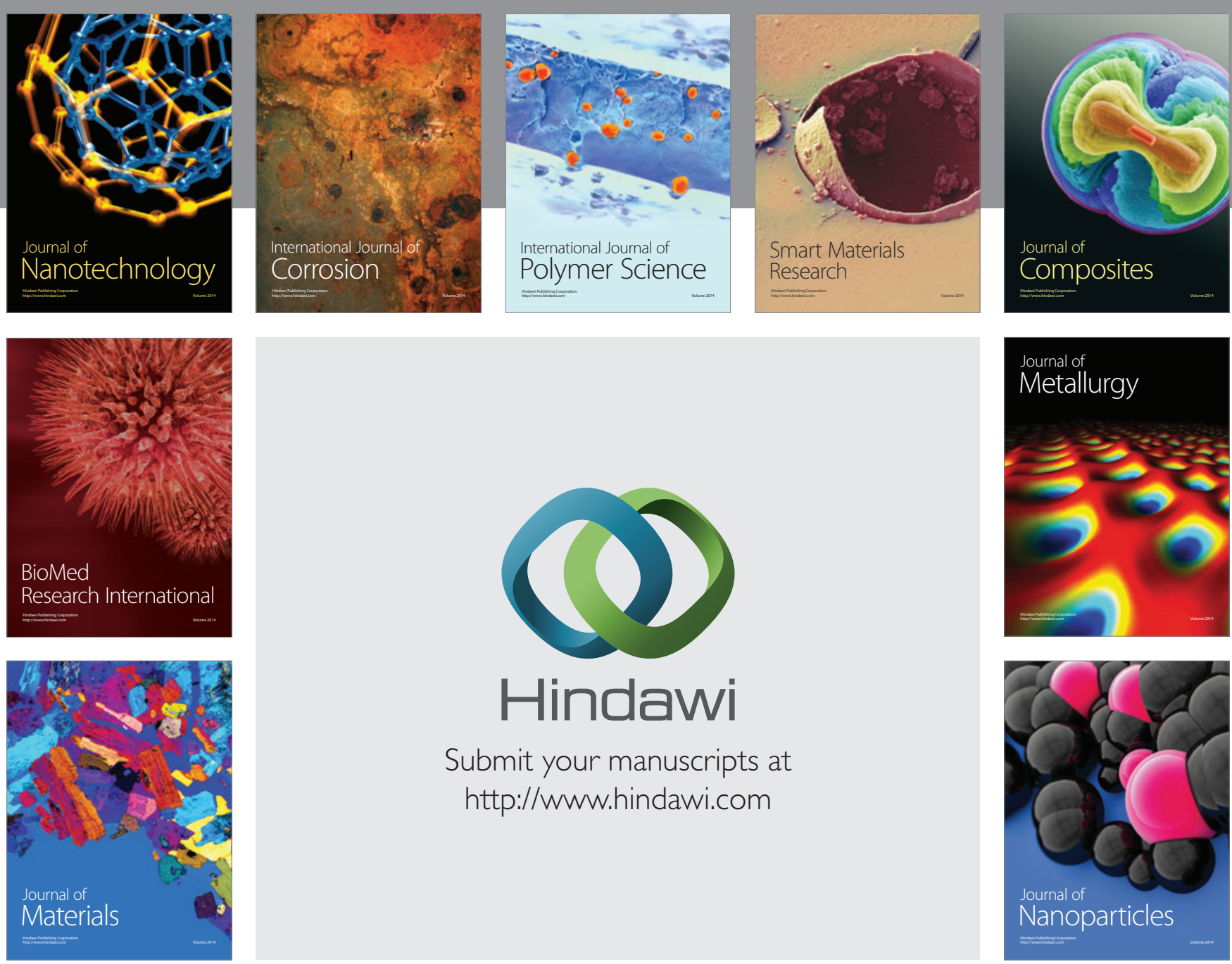

Submit your manuscripts at http://www.hindawi.com
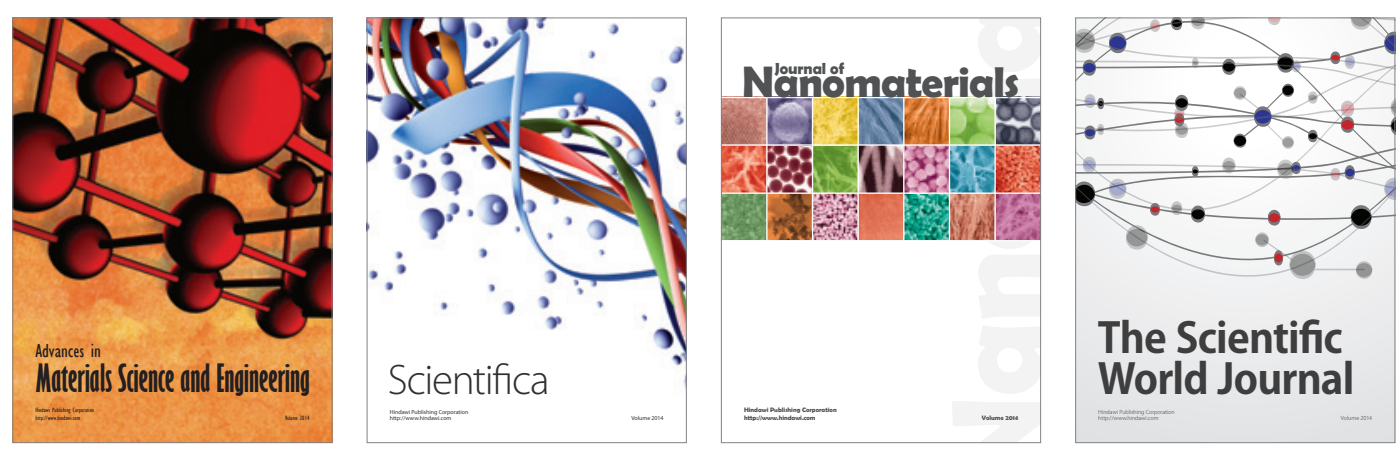

\section{The Scientific World Journal}
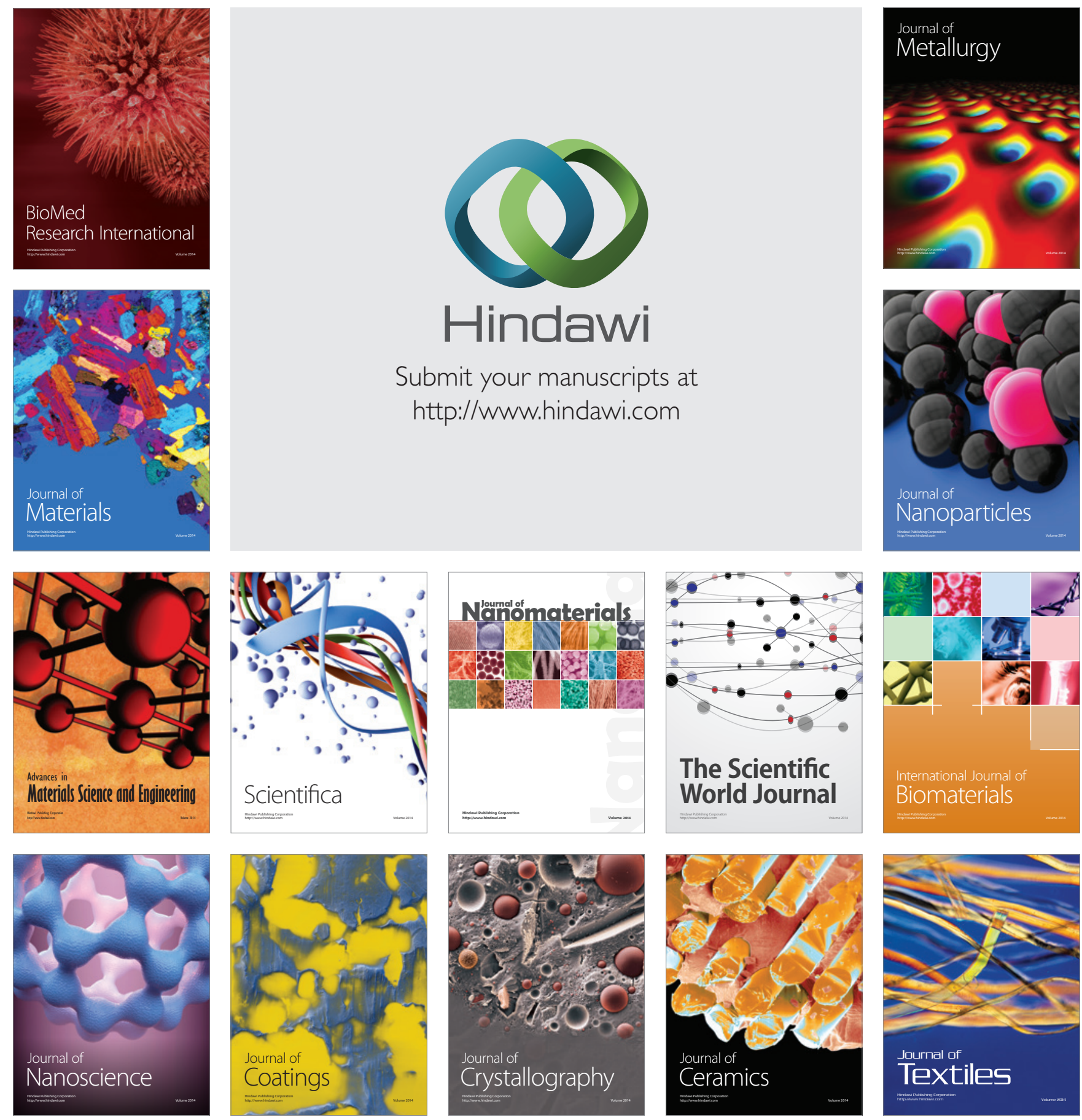